WUB 99-6

RUB-TPII-03/99

\title{
Large-order trend of the anomalous-dimensions spectrum of trilinear twist-3 quark operators
}

\author{
M. Bergmann,, 目 W. Schroers, ${ }^{2}$ 田 and N. G. Stefanis, ${ }^{3}$ 驲 \\ ${ }^{1}$ SerCon, c/o IBM Deutschland GmbH, \\ Karl-Arnold-Platz 1A, \\ D-40774 Düsseldorf, Germany \\ ${ }^{2}$ Fachbereich Physik, \\ Universität Wuppertal, \\ D-42097 Wuppertal, Germany \\ ${ }^{3}$ Institut für Theoretische Physik II, \\ Ruhr-Universität Bochum, \\ D-44780 Bochum, Germany
}

(October 25, 2018)

\begin{abstract}
The anomalous dimensions of trilinear-quark operators are calculated at leading twist $t=3$ by diagonalizing the one-gluon exchange kernel of the renormalization-group type evolution equation for the nucleon distribution amplitude. This is done within a symmetrized basis of Appell polynomials of maximum degree $M$ for $M \gg 1$ (up to order 400) by combining analytical and numerical algorithms. The calculated anomalous dimensions form a degenerate system, whose upper envelope shows asymptotically logarithmic behavior.
\end{abstract}

11.10.Hi, 11.10.Jj, 11.15.Pg, 12.38.Aw

Typeset using REVTEX

\footnotetext{
*E-mail: michael.bergmann@sercon.de

${ }^{\dagger}$ Email: wolfram@theorie.physik.uni-wuppertal.de

${ }^{\ddagger}$ Email: stefanis@tp2.ruhr-uni-bochum.de
} 
The anomalous dimensions of field operators are crucial ingredients in determining the scale dependence of hadronic wave functions in QCD. Though the calculation of the boundstate wave function at the initial (low-momentum) scale cannot be determined within perturbative QCD and requires nonperturbative methods (for a review, see, [1]), its evolution is governed by a renormalization-group type evolution equation [2] which in leading order reduces to the one-gluon-exchange kernel.

Bilinear quark operators of lowest twist $(t=2)$ are representations of the collinear conformal group and therefore their anomalous dimensions (to first order in $\alpha_{\mathrm{s}}$ ) are completely determined by collinear conformal invariance (see, e.g., [3, [.6.5,6]). Therefore, the eigenvalue problem of twist-2 quark operators with an arbitrary number of total derivatives is exactly solvable. In contrast, the anomalous-dimensions matrix of trilinear quark operators of leading twist-3 cannot be diagonalized completely by collinear conformal invariance [7, 8, 9, 10, 11, 12]. As a result, neither the eigenfunctions nor the eigenvalues (alias the anomalous dimensions) of the nucleon evolution equation are known explicitly. Hence one has to diagonalize the anomalous dimensions matrix order by order within an appropriate operator basis.

While operators involving total derivatives do not contribute to forward scattering amplitudes, like deep-inelastic scattering, exclusive processes receive contributions from nonforward matrix elements, and consequently the mixing with trilinear quark operators of twist-3 containing total derivatives cannot be neglected. Given that the baryon (hadron) distribution amplitudes are determined at low normalization scales, of the order of $1 \mathrm{GeV}$ or less, [1,13, 14, 15], the knowledge of anomalous dimensions is indispensable in confronting calculated observables, like the proton form factor, with experimental data at larger values of the momentum transfer.

In this letter we present a systematic treatment of the nucleon evolution equation, focusing on the large-order, i.e., quasi-asymptotic, behavior of the spectrum of the anomalous dimensions of twist-3 multiplicatively renormalizable baryonic $I_{1 / 2}$-operators with the number $M$ of total derivatives acting on these fields tending to infinity. More precisely, we consider operators of the following form

$$
\mathcal{V}_{\gamma}^{\left(n_{1} n_{2} n_{3}\right)}(0) \equiv(i z \cdot D)^{n_{1}} u(0)\left[C \gamma_{\mu} z_{\mu}\right](i z \cdot D)^{n_{2}} u(0)(i z \cdot D)^{n_{3}} \gamma_{5} d_{\gamma}(0)
$$

with $n_{1}+n_{2}+n_{3}=M$, and where $z$ is an auxiliary lightlike vector (i.e., $z^{2}=0$ ) to project out the leading twist contribution [1]. Note that since there are $M+1$ independent operators at each order, one has to diagonalize a $(M+1) \times(M+1)$ matrix in order to determine the corresponding eigenvectors and eigenvalues. This is done here within a basis of trilinear quark operators in terms of appropriately symmetrized Appell polynomials (see below) by analytically diagonalizing the nucleon evolution equation up to order 7 , and proceeding beyond that order up to a maximum order of 400 numerically.

We have already elaborated on these issues in a series of papers [16, [17, 18, 19, 20, 12], and the present work complements and generalizes our previous results, establishing in particular that the upper envelope of the spectrum for very large order $M$ follows a logarithmic law given by the fit

$$
\gamma_{n}(M)=-0.638+0.8882 \ln (M+1.91)
$$

for any member of the spectrum labeled by the index $n$. 
Before proceeding with the presentation of our analysis, let us first mention a very recent alternative approach by Braun and collaborators [21, based on the observation that the eigenvalues of a helicity $\lambda=3 / 2$ baryon, (say, the $\Delta^{++}$) can be determined exactly because the corresponding Hamiltonian $\mathcal{H}_{3 / 2}$ is integrable and can be formally treated as a one-dimensional XXX Heisenberg spin magnet of noncompact spin $s=-1$. Though the Hamiltonian $\mathcal{H}_{1 / 2}$ (relevant for the nucleon) is not integrable and consequently the corresponding eigenvalue problem is not exactly sovable, these authors argue that the helicity-flip terms can be treated as a small perturbation, so that the spectrum of anomalous dimensions can be determined by calculating the mixing matrix elements of these terms in the basis of the exact eigenfunctions of $\mathcal{H}_{3 / 2}$. This is an attractive mathematical framework, and we shall compare our results with theirs at the end of the paper.

Let us now enter our approach in more detail.

The evolution equation is given by [2]

$$
x_{1} x_{2} x_{3}\left[\frac{\partial}{\partial \xi} \tilde{\Phi}\left(x_{i}, Q^{2}\right)+\frac{3}{2} \frac{C_{\mathrm{F}}}{\beta_{0}} \tilde{\Phi}\left(x_{i}, Q^{2}\right)\right]=\frac{C_{\mathrm{F}}}{\beta_{0}} \int_{0}^{1}[d y] V\left(x_{i}, y_{i}\right) \tilde{\Phi}\left(y_{i}, Q^{2}\right)
$$

where

$$
\xi \equiv \frac{\beta_{0}}{4 \pi} \int_{\mu^{2}}^{Q^{2}} \frac{d k_{\perp}^{2}}{k_{\perp}^{2}} \alpha_{\mathrm{s}}\left(k_{\perp}^{2}\right)=\ln \frac{\alpha_{\mathrm{s}}\left(\mu^{2}\right)}{\alpha_{\mathrm{s}}\left(Q^{2}\right)}=\ln \frac{\ln Q^{2} / \Lambda_{\mathrm{QCD}}^{2}}{\ln \mu^{2} / \Lambda_{\mathrm{QCD}}^{2}}
$$

is the evolution "time" parameter, $\beta_{0}=11-\frac{2}{3} n_{\mathrm{f}}=9$ for three flavors, $\Phi=x_{1} x_{2} x_{3} \tilde{\Phi}$ denotes the full nucleon distribution amplitude, $\{\tilde{\Phi}\}$ its eigenfunctions, and $C_{\mathrm{F}}=\left(N_{\mathrm{c}}^{2}-1\right) / 2 N_{\mathrm{c}}$, with the integration measure defined by $[d y]=d y_{1} d y_{2} d y_{3} \delta\left(1-y_{1}-y_{2}-y_{3}\right)$. The kernel $V\left(x_{i}, y_{i}\right)=V\left(y_{i}, x_{i}\right)$ is the sum over one-gluon interactions between quark pairs $\{i, j\}$ at order $\alpha_{\mathrm{s}}$ and is not a function but a distribution from which infrared divergences at $x_{i}=y_{i}$ have been removed.

We propose to solve this evolution equation by employing factorization of the dependence on longitudinal momentum fractions from that on the external (large) momentum scale $Q^{2}$, the latter being controlled by

$$
\frac{\partial}{\partial \xi} \tilde{\Phi}_{n}\left(x_{i}, Q^{2}\right)=-\gamma_{n} \tilde{\Phi}_{n}\left(x_{i}, Q^{2}\right)
$$

with solutions

$$
\tilde{\Phi}_{n}\left(x_{i}, Q^{2}\right)=\tilde{\Phi}_{n}\left(x_{i}\right)\left[\frac{\alpha_{\mathrm{s}}\left(Q^{2}\right)}{\alpha_{\mathrm{s}}\left(\mu^{2}\right)}\right]^{\gamma_{n}} \simeq \tilde{\Phi}_{n}\left(x_{i}\right)\left(\ln \frac{Q^{2}}{\Lambda_{\mathrm{QCD}}^{2}}\right)^{-\gamma_{n}} .
$$

This allows us to write the full nucleon distribution amplitude in the form

$$
\Phi\left(x_{i}, Q^{2}\right) \sim x_{1} x_{2} x_{3} \sum_{n=0}^{\infty} B_{n}\left(\mu^{2}\right) \tilde{\Phi}_{n}\left(x_{i}\right)\left(\ln \frac{Q^{2}}{\Lambda_{\mathrm{QCD}}^{2}}\right)^{-\gamma_{n}}
$$

where $\tilde{\Phi}_{n}\left(x_{i}\right)$ are appropriate but not tabulated polynomials, and the expansion coefficients $B_{n}$ encode the nonperturbative input of the bound-states dynamics at the factorization scale $\mu$. 
From the factorized form of $\tilde{\Phi}_{n}\left(x_{i}, Q^{2}\right)$ in Eq. (6), it follows that the evolution equation for the $x$-dependence reduces to the characteristic equation

$$
x_{1} x_{2} x_{3}\left[\frac{3}{2} \frac{C_{\mathrm{F}}}{\beta_{0}}-\gamma_{n}\right] \tilde{\Phi}\left(x_{i}\right)=\frac{C_{\mathrm{B}}}{\beta_{0}} \int_{0}^{1}[d y] V\left(x_{i}, y_{i}\right) \tilde{\Phi}\left(y_{i}\right)
$$

with $C_{\mathrm{B}}=\left(N_{\mathrm{c}}+1\right) / 2 N_{\mathrm{c}}$.

To proceed, it is convenient to consider the kernel $V\left(x_{i}, y_{i}\right)$ as being an operator expanded on the polynomial basis [2] $\left|x_{1}^{k} x_{3}^{l}\right\rangle \equiv|k l\rangle$ (recall that because of momentum conservation, only two out of three $x_{i}$ variables are linearly independent), i.e., to write

$$
\hat{V} \equiv \int_{0}^{1}[d y] V\left(x_{i}, y_{i}\right)
$$

and convert Eq. (8) into the algebraic equation

$$
\left[\frac{3}{2} \frac{C_{\mathrm{F}}}{\beta_{0}}-2 \frac{C_{\mathrm{B}}}{\beta_{0}} \frac{\hat{V}}{2 w\left(x_{i}\right)}\right] \tilde{\Phi}_{n}\left(x_{i}\right)=\gamma_{n} \tilde{\Phi}_{n}\left(x_{i}\right),
$$

where $w\left(x_{i}\right)=x_{1} x_{2} x_{3}=x_{1}\left(1-x_{1}-x_{3}\right) x_{3}$ is the weight function of the orthogonal basis. In this way, the action of the operator $\hat{V}$ can be completely determined by a matrix, namely:

$$
\frac{\hat{V}|k l\rangle}{2 w\left(x_{i}\right)}=\frac{1}{2} \sum_{i, j}^{i+j \leq M}|i j\rangle U_{i j, k l} .
$$

The corresponding eigenvalues are then determined by the roots $\eta_{n}$ of the characteristic polynomial that diagonalizes the matrix $U$ :

$$
\hat{V} \tilde{\Phi}_{n}\left(x_{i}\right)=-\eta_{n} w\left(x_{i}\right) \tilde{\Phi}_{n}\left(x_{i}\right)
$$

so that the anomalous dimensions are given by

$$
\gamma_{n}(M)=\frac{1}{\beta_{0}}\left(\frac{3}{2} C_{\mathrm{F}}+2 \eta_{n}(M) C_{\mathrm{B}}\right)
$$

with the orthogonalization prescription

$$
\int_{0}^{1}[d x] w\left(x_{i}\right) \tilde{\Phi}_{m}\left(x_{i}\right) \tilde{\Phi}_{n}\left(x_{i}\right)=\frac{1}{N_{m}} \delta_{m n}
$$

where $N_{m}$ are appropriate normalization constants (see Table 1).

Within the basis $|k l\rangle$, the matrix $U$ can be diagonalized to provide eigenfunctions, which are polynomials of degree $M=k+l=0,1,2,3 \ldots$, with $M+1$ eigenfunctions for each $M$. This will be done within a basis of symmetrized Appell polynomials defined by [18, 20, 12,

$$
\begin{aligned}
\tilde{\mathcal{F}}_{m n}\left(x_{1}, x_{3}\right) & =\frac{1}{2}\left[\mathcal{F}_{m n}\left(x_{1}, x_{3}\right) \pm \mathcal{F}_{n m}\left(x_{1}, x_{3}\right)\right] \\
& =\sum_{k, l=0}^{k+l \leq m+n} Z_{k l}^{m n}|k l\rangle
\end{aligned}
$$


where + refers to the case $m \geq n$ and - to the case $m<n$, and the Appell polynomials are defined in terms of special hypergeometric functions, according to

$$
\mathcal{F}_{m n}\left(x_{1}, x_{3}\right) \equiv \mathcal{F}_{m n}^{(M)}\left(5,2,2 ; x_{1}, x_{3}\right)
$$

These polyomials constitute an orthogonal set on the triangle $T=T\left(x_{1}, x_{3}\right)$ with $x_{1}>0$, $x_{3}>0, x_{1}+x_{3}<1$ and provide a very suitable basis to solve the eigenvalue equation for the nucleon because $\hat{V}$ (i) is blockdiagonal for different polynomial orders, and (ii) commutes with the permutation operator $P_{13}=[321]$, i.e., $\left[P_{13}, \hat{V}\right]=0$. Hence $\hat{V}$ becomes, in addition, blockdiagonal within each sector of permutation-symmetry eigenfunctions at fixed order $M$. As a result, the kernel $\hat{V}$ can be analytically diagonalized up to order seven, providing a total of $n_{\max }(M)=\frac{1}{2}(M+1)(M+2)=36$ eigenvectors and eigenvalues (alias eigenfunctions and anomalous dimensions). Beyond that order, the evolution equation has to be solved numerically because the roots of the characteristic polynomial of matrices with rank four cannot be determined analytically.

From the practical point of view, such a large set of eigenfunctions surely exceeds the number of theoretical constraints on the nonperturbative input coefficients $B_{n}$ that can be derived from QCD sum rules [1, 22, 23], lattice simulations [24, or by fitting experimental data [25]. However, yet much larger orders are necessary in order to extract the asymptotic behavior of the anomalous-dimensions spectrum.

For this purpose, let us express all nucleon eigenfunctions as linear combinations of symmetrized Appell polynomials of the same order $M$

$$
\tilde{\Phi}_{k}\left(x_{i}\right)=\sum_{m, n=0}^{m+n=M} c_{m n}^{k} \mathcal{F}_{m n}\left(5,2,2 ; x_{1}, x_{3}\right)
$$

and rearrange $\tilde{\mathcal{F}}_{m n}$ (cf. Eq. (15)), which belongs to a definite symmetry class $S_{n}= \pm 1$ within order $M$, in the form of an (arbitrary) vector to read

$$
\tilde{\mathcal{F}}_{m n}\left(x_{1}, x_{3}\right) \longmapsto \tilde{\mathcal{F}}_{q}\left(x_{1}, x_{3}\right) \text {. }
$$

Then Hilbert-Schmidt orthogonalization yields a basis

$$
\left|\tilde{\mathcal{F}}_{q}^{\prime}\right\rangle=\sum_{k, l} Z_{k l}^{q}|k l\rangle
$$

with

$$
\int_{0}^{1}[d x] w\left(x_{i}\right) \tilde{\mathcal{F}}_{q}^{\prime} \tilde{\mathcal{F}}_{q^{\prime}}^{\prime} \propto \delta_{q q^{\prime}}
$$

so that

$$
\frac{\hat{V}\left|\tilde{\mathcal{F}}_{q}^{\prime}\right\rangle}{2 w\left(x_{i}\right)}=\frac{1}{2} \sum_{i, j, k, l} Z_{k l}^{q} U_{i j, k l}|i j\rangle .
$$

Note that the construction of polynomials depending on two variables via the HilbertSchmidt method has no unique solution, but depends on the order in which the orthogonalization is performed. Since beyond order $M=3$, neither the eigenvalues nor the 
normalization factors are rational numbers, one has to find which representation is more convenient for calculations.

The last step in determining the eigenfunctions and eigenvalues of $\hat{V}$ is to define the matrix

$$
\mathcal{M}_{q^{\prime} q}=\int_{0}^{1}[d x] w\left(x_{1},\left(1-x_{1}-x_{3}\right), x_{3}\right) \tilde{\mathcal{F}}_{q^{\prime}}^{\prime}\left(x_{1}, x_{3}\right) \hat{V}\left(x_{1}, x_{3}\right) \tilde{\mathcal{F}}_{q}^{\prime}\left(x_{1}, x_{3}\right)
$$

and calculate the roots of the characteristic polynomial

$$
\mathcal{P}(\eta)=\operatorname{det}\left[\mathcal{M}_{q^{\prime} q}-\eta I_{q^{\prime} q}\right]
$$

Consequently, in terms of the eigenvectors $\boldsymbol{m}_{q}=\left(m_{q}^{1}, \ldots m_{q}^{q^{\prime}}\right)$ of $\mathcal{M}_{q^{\prime} q}$, the eigenfunctions of the evolution equation are given by

$$
\begin{aligned}
\tilde{\Phi}_{q}\left(x_{1}, x_{3}\right) & \propto \sum_{q^{\prime}} m_{q}^{q^{\prime}} \tilde{\mathcal{F}}_{q^{\prime}}^{\prime}\left(x_{1}, x_{3}\right) \\
& =\sum_{k, l} a_{k l}^{q}|k l\rangle .
\end{aligned}
$$

For every order $M$, there are $M+1$ eigenfunctions of the same order with an excess of symmetric terms by one for even orders. A compedium of the results up to order $M=4$, yielding a total of 15 eigenfunctions and associated anomalous dimensions, is given in Table 1 . The precision of orthogonality is at least $10^{-8}$.

It turns out that the eigenfunctions $\left\{\tilde{\Phi}_{n}\right\}$ of the nucleon evolution equation satisfy a commutative algebra subject to the triangular condition $|\mathcal{O}(k)-\mathcal{O}(l)| \leq \mathcal{O}(m) \leq \mathcal{O}(k)+$ $\mathcal{O}(l):$

$$
\tilde{\Phi}_{k}\left(x_{i}\right) \tilde{\Phi}_{l}\left(x_{i}\right)=\sum_{m=0}^{\infty} F_{k l}^{m} \tilde{\Phi}_{m}\left(x_{i}\right)
$$

with structure coefficients $F_{k l}^{m}$ given by

$$
F_{k l}^{m}=N_{m} \int_{0}^{1}[d x] x_{1} x_{3}\left(1-x_{1}-x_{3}\right) \tilde{\Phi}_{m}\left(x_{i}\right) \tilde{\Phi}_{k}\left(x_{i}\right) \tilde{\Phi}_{l}\left(x_{i}\right)
$$

$\mathcal{O}(k)$ being defined by

$$
\mathcal{O}(k)= \begin{cases}0 & k=0 \\ 1 & 1 \leq k \leq 2 \\ 2 & 3 \leq k \leq 5 \\ 3 & 6 \leq k \leq 9 \\ 4 & k=10,11 \\ \vdots & \cdots\end{cases}
$$

Note that the structure coefficients are symmetric, i.e., $F_{k l}^{m}=F_{l k}^{m}$. Furthermore, $F_{k k}^{0}=\frac{N_{0}}{N_{k}}$. The utility of this algebra derives from the fact that once the structure coefficients have been computed, they can be used to express any function $f\left(x_{1}, x_{3}\right)$ in terms of the nucleon eigenfunctions. The values of $F_{l k}^{m}$ up to $\mathcal{O}(k)=11$ are tabulated in [18. Unfortunately, it is 
Table 1. Orthogonal eigenfunctions $\tilde{\Phi}_{n}\left(x_{i}\right)=\sum_{k l} a_{k l}^{n} x_{1}^{k} x_{3}^{l}$ of the nucleon evolution equation up to order $M=4$ in terms of the coefficient matrix $a_{k l}^{n}\left(a_{k l}^{n}=S_{n} a_{l k}^{n}\right.$ with $n$ fixed) and the corresponding anomalous dimensions $\gamma_{n}(M)$ defined in the text. The numerical results for $n \geq 12$ have been obtained with a much higher numerical accuracy than shown in this table.

\begin{tabular}{|c|c|c|c|c|c|c|c|c|}
\hline$n$ & $M$ & $S_{n}$ & $\gamma_{n}$ & \multicolumn{2}{|c|}{$\eta_{n}$} & \multicolumn{2}{|l|}{$N_{n}$} & $a_{00}^{n}$ \\
\hline 0 & 0 & 1 & $\frac{2}{27}$ & \multicolumn{2}{|c|}{-1} & \multicolumn{2}{|c|}{120} & 1 \\
\hline 1 & 1 & -1 & $\frac{26}{81}$ & \multicolumn{2}{|c|}{$\frac{2}{3}$} & \multicolumn{2}{|c|}{1260} & 0 \\
\hline 2 & 1 & 1 & $\frac{10}{27}$ & \multicolumn{2}{|c|}{1} & \multicolumn{2}{|c|}{420} & -2 \\
\hline 3 & 2 & 1 & $\frac{38}{81}$ & & $\frac{5}{3}$ & \multicolumn{2}{|l|}{756} & 2 \\
\hline 4 & 2 & -1 & $\frac{46}{81}$ & & $\frac{7}{3}$ & \multicolumn{2}{|c|}{34020} & 0 \\
\hline 5 & 2 & 1 & $\frac{16}{27}$ & & $\frac{5}{2}$ & \multicolumn{2}{|c|}{1944} & 2 \\
\hline 6 & 3 & 1 & $\frac{115-\sqrt{97}}{162}$ & \multicolumn{2}{|c|}{$\frac{1}{24}$} & \multicolumn{2}{|c|}{$\frac{4620(485+11 \sqrt{97})}{97}$} & 1 \\
\hline 7 & 3 & 1 & $\frac{115+\sqrt{97}}{162}$ & \multicolumn{2}{|c|}{$\frac{79+\sqrt{97}}{24}$} & \multicolumn{2}{|c|}{$4620(485-11 \sqrt{97})$} & 1 \\
\hline 8 & 3 & -1 & $\frac{559-\sqrt{4801}}{810}$ & \multicolumn{2}{|c|}{$\frac{-(-379+\sqrt{4801})}{120}$} & \multicolumn{2}{|c|}{$\frac{27720(33607-247 \sqrt{4801})}{4801}$} & 0 \\
\hline 9 & 3 & -1 & $\frac{559+\sqrt{4801}}{810}$ & \multicolumn{2}{|c|}{$379+\sqrt{4801}$} & \multicolumn{2}{|c|}{$\begin{array}{c}4801 \\
27720(33607+247 \sqrt{4801})\end{array}$} & 0 \\
\hline 10 & 4 & -1 & $\frac{346-\sqrt{1081}}{405}$ & \multicolumn{2}{|c|}{$\frac{-(-256+\sqrt{1081})}{60}$} & \multicolumn{2}{|c|}{$196560(7567-13 \sqrt{1081})$} & 0 \\
\hline 11 & 4 & -1 & $\frac{346+\sqrt{1081}}{405}$ & \multicolumn{2}{|c|}{$256+\sqrt{1081}$} & \multicolumn{2}{|c|}{$196560(7567+13 \sqrt{1081})$} & 0 \\
\hline 12 & 4 & 1 & 0.70204 & \multicolumn{2}{|c|}{$\frac{60}{3.23876}$} & \multicolumn{2}{|c|}{1081} & 153.37061 \\
\hline 13 & 4 & 1 & 0.80651 & \multicolumn{2}{|c|}{3.94397} & 1 & & 332.500864 \\
\hline 14 & 4 & 1 & 0.93589 & 4.8 & 727 & 1 & & -137.11538 \\
\hline$n$ & $a_{10}^{n}$ & $a_{20}^{n}$ & $a_{11}^{n}$ & $a_{30}^{n}$ & $a_{21}^{n}$ & $a_{40}^{n}$ & $a_{31}^{n}$ & $a_{22}^{n}$ \\
\hline 0 & 0 & 0 & 0 & 0 & 0 & 0 & 0 & 0 \\
\hline 1 & 1 & 0 & 0 & 0 & 0 & 0 & 0 & 0 \\
\hline 2 & 3 & 0 & 0 & 0 & 0 & 0 & 0 & 0 \\
\hline 3 & -7 & 8 & 4 & 0 & 0 & 0 & 0 & 0 \\
\hline 4 & 1 & $-\frac{4}{3}$ & 0 & 0 & 0 & 0 & 0 & 0 \\
\hline 5 & -7 & $\frac{14}{3}$ & 14 & 0 & 0 & 0 & 0 & 0 \\
\hline 6 & -6 & $\frac{41+\sqrt{97}}{4}$ & $\frac{3(31-\sqrt{97})}{4}$ & $\frac{-5(17+\sqrt{97})}{16}$ & $\frac{-5(31-\sqrt{97})}{8}$ & 0 & 0 & 0 \\
\hline 7 & -6 & $\frac{41-\sqrt{97}}{4}$ & $\frac{3(31+\sqrt{97})}{4}$ & $\frac{-5(17-\sqrt{97})}{16}$ & $\frac{-5(31+\sqrt{97})}{8}$ & 0 & 0 & 0 \\
\hline 8 & 1 & -3 & 0 & $\frac{601+\sqrt{4801}}{264}$ & $\frac{59-\sqrt{4801}}{44}$ & 0 & 0 & 0 \\
\hline 9 & 1 & -3 & 0 & $\frac{601-\sqrt{4801}}{264}$ & $\frac{59+\sqrt{4801}}{44}$ & 0 & 0 & 0 \\
\hline 10 & 1 & -5 & 0 & $\frac{379+\sqrt{1081}}{48}$ & $\frac{61-\sqrt{1081}}{8}$ & $\frac{-(159+\sqrt{1081})}{40}$ & $\frac{-(61-\sqrt{1081})}{8}$ & 0 \\
\hline 11 & 1 & -5 & 0 & $\frac{379-\sqrt{1081}}{48}$ & $\frac{61+\sqrt{1081}}{8}$ & $\frac{-(159-\sqrt{1081})}{40}$ & $\frac{-(61+\sqrt{1081})}{8}$ & 0 \\
\hline 12 & -1380.33552 & 5232.86956 & 5006.42414 & -8063.85349 & -9178.44426 & 4345.63139 & 4926.80699 & 8503.27454 \\
\hline 13 & -2992.50778 & 9240.51876 & 17166.06044 & -11695.76593 & -31471.11081 & 5068.49438 & 19489.65169 & 23962.91822 \\
\hline 14 & 1234.03849 & -1843.05428 & -12981.41464 & -587.61051 & 23799.26017 & 1382.85660 & -10302.90296 & -26992.71442 \\
\hline
\end{tabular}




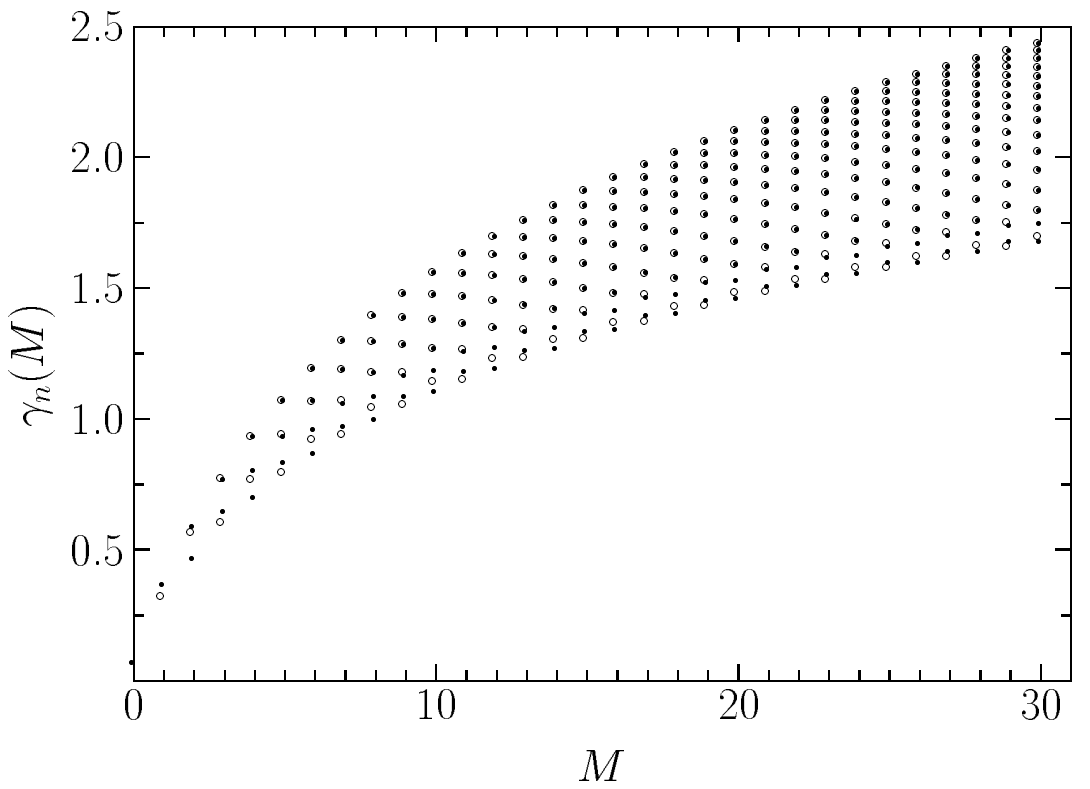

Figure 1. Illustration of the spectrum of the anomalous dimensions up to order $M=30$, comprising symmetric (full circles) and antisymmetric (open circles) contributions.

not that easy to identify the proper group (and physical symmetry) underlying this algebra. Work in this direction is still in progress.

Next, we present the results obtained for the anomalous-dimensions spectrum. An impression of the detailed structure of the spectrum is provided by fig. 1. Both symmetry classes under the permutation $P_{13}$ are shown: open circles stand for values belonging to $S_{n}=-1$ (antisymmetric sector) and black dots for those belonging to $S_{n}=1$ (symmetric sector). As the order $M$ increases, the degeneracy of the spectrum also increases because more and more operators with the same quantum numbers contribute and the density of eigenvalues becomes very high. Because all $\gamma_{n}$ are positive fractional numbers increasing with the counting index $n$, higher terms in the eigenfunctions decomposition of the nucleon distribution amplitude are gradually suppressed (cf. Eq. 7).

At very large order a different picture for the large-order behavior of the spectrum of anomalous dimensions develops, namely one of logarithmic rise. Indeed, while the low-order spectrum with $M \leq 3$ [2, [7,8,9] can be reproduced by the empirical power-law $\gamma_{n}(M)=0.37 M^{0.565}$, the inclusion of large orders seems to be better described by a logarithm. Extending the calculation up to a maximum order of $M=400$, we finally obtain the spectrum displayed in fig. 2.

The anomalous-dimensions spectrum can be reproduced by the logarithmic fit

$$
\gamma_{n}(M)=c+d \ln (M+b)
$$

Up to order 150 both sectors of eigenvalues are included, i.e., $S_{n}= \pm 1$. Beyond that order, for reasons of technical convenience, only the antisymmetric ones have been taken into account. However, since the spacings become very dense asymptotically, this poses no restrictions on the validity of the calculation. The upper envelope of the spectrum is best described by the following values of the parameters with their errors: $b=1.90989 \pm 0.00676$, 


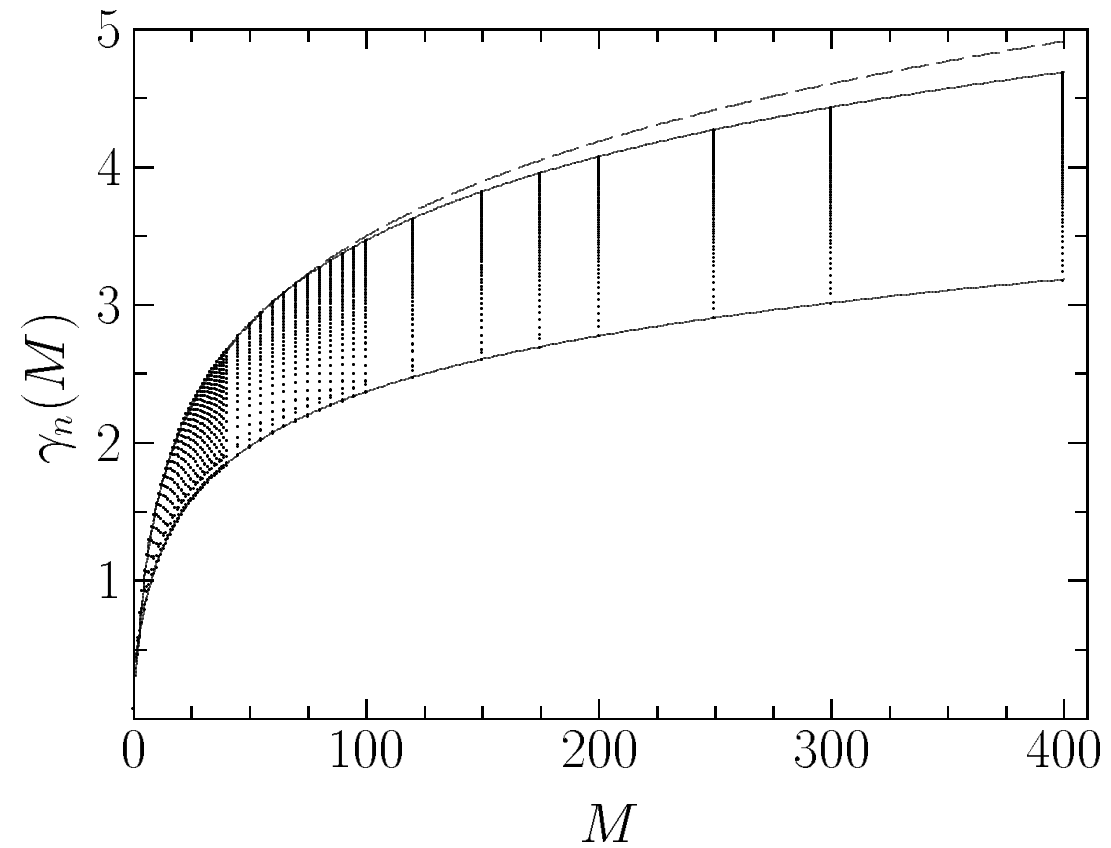

Figure 2. Spectrum of the anomalous dimensions of trilinear twist-3 quark operators up to order $M=400$. The solid lines (upper and lower envelopes of the spectrum) represent logarithmic fits up to the maximum considered order 400, taking into consideration all orders above 10. The dashed line gives for comparison a previous logarithmic fit [12] (see text) which takes into account all orders up to 150.

$c=-0.637947 \pm 0.000634$, and $d=0.88822 \pm 0.000119$. For the lower envelope, the corresponding values are $b=3.006 \pm 0.483, c=-0.3954 \pm 0.0290$, and $d=0.59691 \pm 0.00545$. The spacing of eigenvalues at very large order is reproduced by the values $b=-0.027 \pm 0.728$, $c=-0.2460 \pm 0.0248$, and $d=0.291883 \pm 0.00475$. It is worth remarking that the previous logarithmic fit $\gamma_{n}(M)=\left[\log _{10}(2.13 M+1.4)\right]^{1.48}$, given in [12], which takes into account all orders up to 150, deviates from that in Eq. (28) only by an amount less than $5 \%$ at order 400. Hence, we conclude that the calculated spectrum shows already asymptotic behavior, rendering the inclusion of still higher orders superfluous. In particular, as one observes from fig. [3, the exponent $d$ for the upper envelope shows scaling behavior for $M \geq 200$, approaching fast the value 0.8882 . Physically, the logarithmic rise of the spectrum is due to the enhanced emission of soft gluons, reflecting the fact that the probability for finding bare quarks decreases [26].

Let us now turn to the recent work by Braun et al. [21]. These authors claim that the lowest two eigenvalues of the spectrum decouple, being separated from the others by a gap, which they interpret as resulting from the formation of a diquark state. Let us see whether our exact large-order calculation confirms their finding. Fig. 6 shows the gap at each particular order between the lowest two anomalous dimensions and the 3rd one as a function of the order $M$ (marked by black dots) in comparison with the gap between the 7th and 8th anomalous dimensions (denoted by crosses), which we found to be the largest gap after the 3rd one. One observes that indeed beyond order 50, the gap of the two lowest anomalous dimensions remains constant (up to the maximum considered order 150), whereas the gap of the higher ones decreases logarithmically like $0.267133 / \ln M$ (solid line), where 


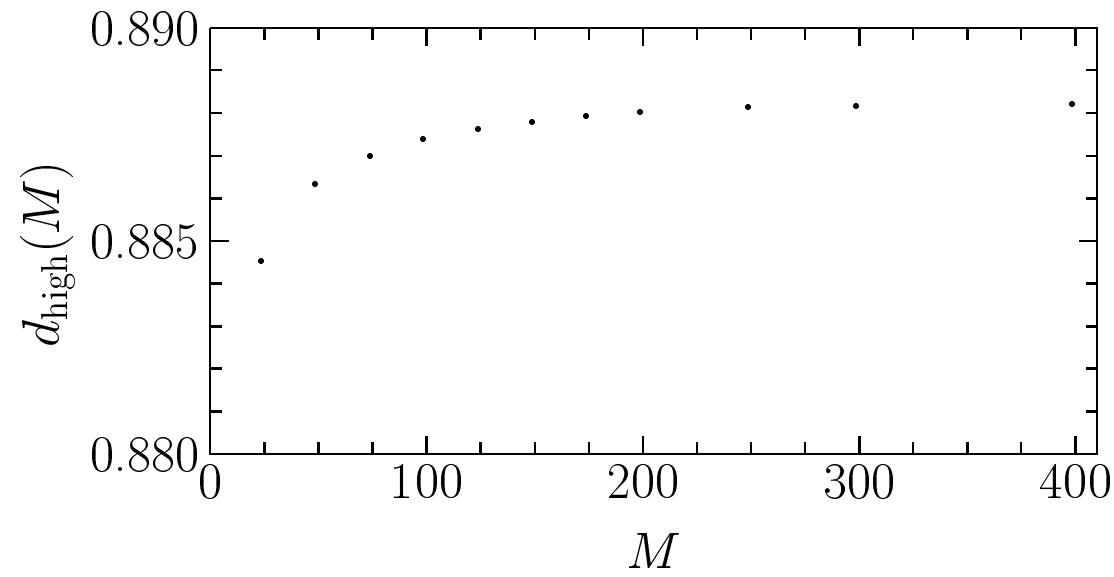

Figure 3. Determination of the exponent $d$ for the upper branch of the anomalous-dimensions spectrum.

the first four shown values were fitted. Note that beyond order 200 only the antisymmetric values were used, so that the calculated gaps in this sector lie slightly higher than the gaps between the combined set of values of anomalous dimensions. This behavior can indeed be interpreted as supporting the results of [21], though our exact calculation gives for that gap

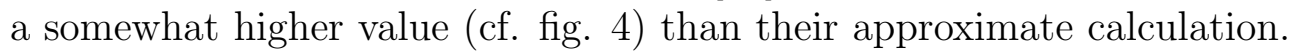

In summary, we have provided convincing evidence that the large-order behavior of the anomalous-dimensions spectrum of trilinear twist-3 quark operators with total derivatives tends to grow logarithmically with order. The exponent for the upper envelope of the spectrum was determined with high precision and found to have the asymptotic value 0.8882. The lowest two levels of the spectrum seem to be separated from higher ones by a gap as found by Braun et al. [21] - possibly indicating the formation of a binary quark system (diquark cluster) inside the nucleon. Yet, scepticism remains whether this finding will survive the inclusion to the kernel of the nucleon evolution equation of higher-order contributions which comprise gluon self-interactions. We have presented explicit results for a set of 15 orthonormalized eigenfunctions of the nucleon evolution equation, employing a symmetrized basis of Appell polynomials, whereas still higher states can be readily constructed by the means developed and used in this work. These results suggest that the objections raised in [21] against the use of Appell polynomials in solving the nucleon evolution equation are not really justified.

\section{Acknowledgments}

We would like to thank Dieter Müller, Pavel Pobylitsa, Maxim Polyakov, and Prof. Grigoris Tsagas for useful discussions. 


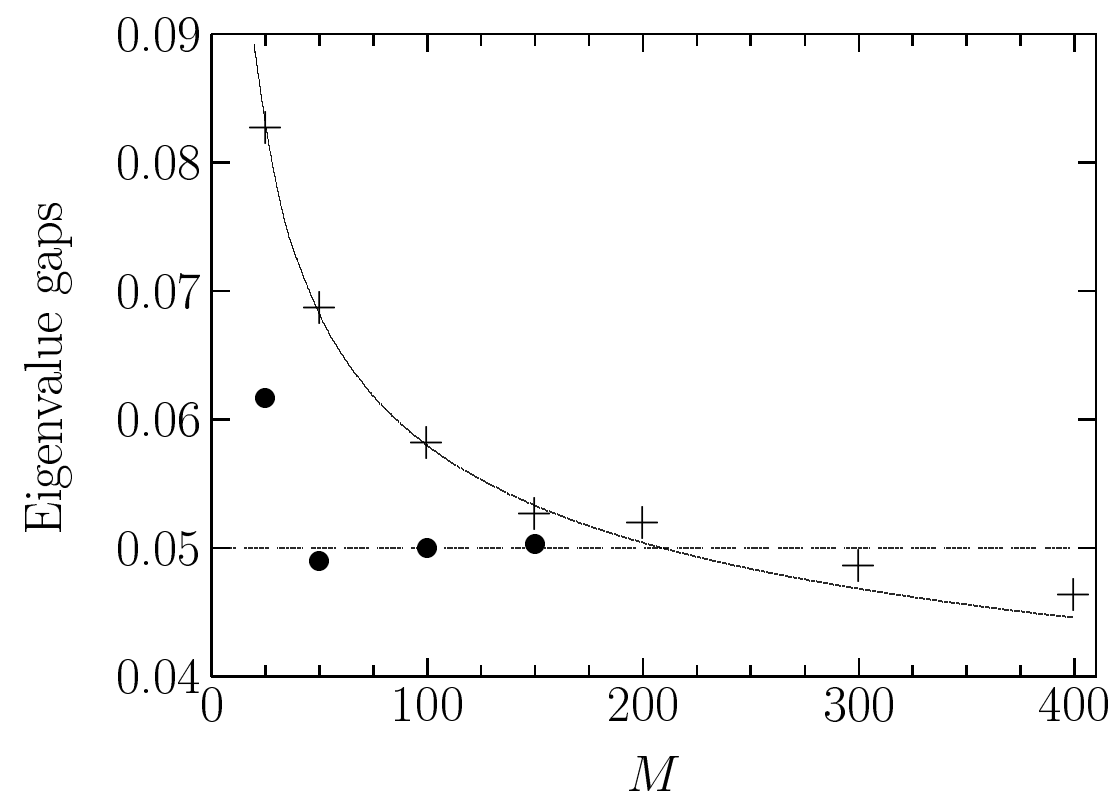

Figure 4. Gaps of eigenvalues in the spectrum of anomalous dimensions.

\section{References}

[1] V.L. Chernyak and A.R. Zhitnitsky, Phys. Rep. 112 (1984) 173.

[2] G.P. Lepage and S.J. Brodsky, Phys. Rev. D 22 (1980) 2157.

[3] A.V. Efremov and A.V. Radyushkin, Phys. Lett. B 94 (1980) 245.

[4] S.J. Brodsky et al., Phys. Lett. B 91 (1980) 239; S.J. Brodsky, Y. Frishman, and G.P. Lepage, Phys. Lett. B 167 (1986) 347; S.J. Brodsky et al., Phys. Rev. D 33 (1986) 1881.

[5] Th. Ohrndorf, Nucl. Phys. B 186 (1981) 153.

[6] D. Müller, Phys. Rev. D 49 (1994) 2525; ibid. D 51 (1995) 3855; hep-ph/9812490; A.V. Belitsky and D. Müller, Nucl. Phys. B 537 (1999) 397.

[7] M. Peskin, Phys. Lett. B 88 (1979) 128.

[8] M. Kremer, Nucl. Phys. B 168 (1979) 272.

[9] K. Tesima, Phys. Lett. B 110 (1981) 319; Nucl. Phys. B 202 (1982) 523.

[10] Th. Ohrndorf, Nucl. Phys. B 198 (1982) 26.

[11] S.-L. Nyeo, Z. Phys. C 54 (1992) 615.

[12] N.G. Stefanis, Dr. nauk (sci.) thesis, JINR, Dubna, 1997, Dubna Report No. 2-97-144, 30pp. (in Russian); Bochum Report No. RUB-TPII-20/96, 1996, 173pp (unpublished).

[13] N.G. Stefanis, Phys. Rev. D 40 (1989) 2305; D 44 (1991) 1616(E).

[14] M. Bergmann and N. G. Stefanis, Phys. Rev. D 48 (1993) R2990.

[15] V.Yu. Petrov et al., hep-ph/9807229.

[16] M. Bergmann and N.G. Stefanis, in: Proc. Workshop on Exclusive Reactions at High Momentum Transfer, Elba, Italy, 24-26 June, 1993, eds. C.E. Carlson, P. Stoler, and M. Taiuti (World Scientific, Singapore, 1994) p. 146.

[17] N.G. Stefanis and M. Bergmann, in: Proc. Workshop on Quantum Field Theoretical Aspects of High Energy Physics, Kyffhäuser, Germany, September 20-24, 1993, eds. B. Geyer and E.-M. Ilgenfritz, p. 112. 
[18] M. Bergmann, Dr. rer. nat. thesis, Bochum University, January 1994, in German (unpublished).

[19] M. Bergmann and N.G. Stefanis, in: International Conference on Quark Confinement and the Hadron Spectrum, Villa Olmo, Como, Italy, 20-24 June, 1994, eds. N. Brambilla and G.M. Prosperi (World Scientific, Singapore, April 1995) p. 269.

[20] N.G. Stefanis, Acta Phys. Pol. B 25 (1994) 1777.

[21] V.M. Braun et al., hep-ph/9902375.

[22] I.D. King and C.T. Sachrajda, Nucl. Phys. B 279 (1987) 785.

[23] V.L. Chernyak, A.A. Ogloblin, and I.R. Zhitnitsky, Z. Phys. C 42 (1989) 583.

[24] D.G. Richards, C.T. Sachrajda, and C.J. Scott, Nucl. Phys. B 286 (1987) 683; G. Martinelli and C.T. Sachrajda, Phys. Lett. B 217 (1989) 319.

[25] J. Bolz and P. Kroll, Z. Phys. A 356 (1996) 327.

[26] Yu.L. Dokshitzer, D.I. Dyakonov, and S.I. Troyan, Phys. Rep. 58 (1980) 269. 